\title{
Complete genome sequence of a marine roseophage provides evidence into the evolution of gene transfer agents in alphaproteobacteria
}

\author{
Sijun Huang ${ }^{1,2 \dagger}$, Yongyu Zhang ${ }^{1,3 \dagger}$, Feng Chen ${ }^{2}$, Nianzhi Jiao ${ }^{1 *}$
}

\begin{abstract}
Roseophage RDJLФ1 is a siphovirus isolated from South China Sea on Roseobacter denitrificans OCh114. Its virion encapsulates $62.7 \mathrm{~kb}$ genome that encodes 87 gene products. RDJL $\Phi 1$ shares similar genome organization and gene content with the marine bacteriophage $\Phi J \mathrm{~L} 001$ and Pseudomonas phages YuA and M6, which are different from those of typical $\lambda$ - or Mu-like phages. Four hallmark genes (ORFs 81 to 84) of RDJLФ1 were highly homologous to RcGTA-like genes 12 to 15. The largest gene (ORF 84) was predicted to encode a tail fibre protein that could be involved in host recognition. Extended phylogenetic and comparative genomic analyses based on 77 RcGTA-like element-containing bacterial genomes revealed that RcGTA-like genes 12 to 15 together appear to be a conserved modular element that could also be found in some phage or prophage genomes. Our study suggests that RcGTA-like genes-containing phages and prophages and complete RcGTAs possibly descended from a same prophage ancestor that had diverged and then evolved vertically. The complete genome of RDJLФ1 provides evidence into the hypothesis that extant RcGTA may be a prophage remnant.
\end{abstract}

\section{Findings}

Viruses are the most abundant entities of the world's oceans, ranging from $\sim 3 \times 10^{6}$ to $\sim 10^{8}$ viruses per $\mathrm{ml}[1]$. Bacteriophages (viruses that infect bacteria) are known to play an important role in regulating the species composition of bacteria [1-4] and in the host evolution through phage-mediated horizontal gene transfer [5-7]. Bacteria in the Roseobacter clade (roseobacter hereafter) are abundant, and typically comprise $10-20 \%$ of marine bacterial communities $[8,9]$. More than 30 genomes of representative roseobacters have been sequenced [10], and the genomics studies also showed that nearly all roseobacter genomes carry a conserved gene transfer agent (GTA) gene cluster of RcGTA (GTA producted by Rhodobacter capsulatus) type [10-14] which could assemble a phage-like particle that transfers random pieces of genome DNA from producing cells to recipient cells through a generalized transduction-like process [15]. The widespread occurrence of RcGTA was

\footnotetext{
* Correspondence: jiao@xmu.edu.cn

† Contributed equally

'State Key Laboratory of Marine Environmental Science, Xiamen University,

Xiamen 361005, PR China

Full list of author information is available at the end of the article
}

hypothesized to be a potential efficient mechanism for horizontal gene transfer [13].

Up to date, only a limited number of roseophage (phage that infects roseobacter) genomes have been reported [16-18], which were all in Podoviridae family. In this study, we presented the genome sequence of Roseophage RDJLФ1 that infects Roseobacter denitrificans OCh114. RDJLФ1 was isolated from the South China Sea surface water $\left(17.597^{\circ} \mathrm{N}, 116.029^{\circ} \mathrm{E}\right)$ collected in September 2007 as previously described [19]. RDJLФ1 was characterized as a host-specific siphovirus, which has an isometric head and a long, flexible, non-contractile tail, and belongs to Siphoviridae family, Caudovirales order [19]. RDJLФ1 is a lytic phage with burst size of $c a .203$ and latent period of $c a .80 \mathrm{~min}$ [19]. This is the first presented genome of a siphovirus infecting marine Roseobacter.

The circularly assembled genome of RDJLФ1 comprises $62,668 \mathrm{bp}$, with a $\mathrm{G}+\mathrm{C}$ content of $57.9 \%$, strongly resembling the $\mathrm{G}+\mathrm{C}$ average (58.0\%) of its host. The whole genome was sequenced by using shotgun library method with 7 -fold coverage. In total, 87 open reading frames (ORFs) were predicted from the genome using Glimmer [20] and GeneMark [21] (Additional file 1, 
Figure 1). No tRNA sequences were identified using the tRNAscan-SE program [22]. Fifty-five gene products have homologous sequences in NCBI non-redundant protein database, whereas only 24 of them have predicted functions. Thirty-eight ORFs are homologous to genes in bacteriophages. Among them, 15 ORFs were homologues of genes in another siphovirus DJL001 that infects an uncharacterized marine sponge-associated alphaproteobacterium, JL001 [23] (Figure 1, indicated by grey shadows). Most of these homologues between RDJLФ1 and JLL01 scatter in similar loci of the genomes. Based on the sequence homology, RDJL $\Phi 1$ is most closely related to $\Phi J$ L001 among all the known phage genomes. Moreover, 7 of those 15 ORFs are also homologous to genes from Pseudomonas phage YuA [24] and/or M6 [25]; YuA and M6 are 91\% identical to each other at the DNA level [24]. Five phage structural proteins, which are tail fibre protein (gp84), tail tape measure protein (gp80), major tail protein (gp78), major capsid protein (gp74) and an unknown structural protein (gp67), could be assigned based on the previously reported proteome analysis (Table 1; the five proteins
Table 1 Phage RDJL $\Phi 1$ structural protein assignment based on the previously reported SDS-PAGE analysis [19]

\begin{tabular}{llll}
\hline SDS-PAGE band & MW $(\mathbf{k D a})$ & ORF no. & Predicted product \\
\hline A & 147.6 & 84 & tail fibre \\
B & 107.9 & 80 & tail tape measure protein \\
C & 53 & 78 & major tail protein \\
D & 52.7 & 67 & unknown structural protein \\
E & 37.7 & 74 & major capsid protein \\
\hline
\end{tabular}

SDS-PAGE: sodium dodecyl sulfate-polyacrylamide gel electrophoresis; MW: molecular weight.

and corresponding SDS-PAGE bands were predicted based on comparing their molecular weights and bands' staining intensity) [19].

The RDJLФ1 genome can be divided into three modules: (i) DNA metabolism and replication, (ii) phage-host interaction, (iii) DNA packaging and structure formation (Figure 1). This type of genome organization is similar to those of $\Phi J L 001, \mathrm{YuA}$ and M6, but differs from those of $\lambda$ - and $\mathrm{Mu}$-like phages [24]. RDJLФ1, ФJL001, YuA and M6 also share the similar gene contents in the three modules. No lysis genes were

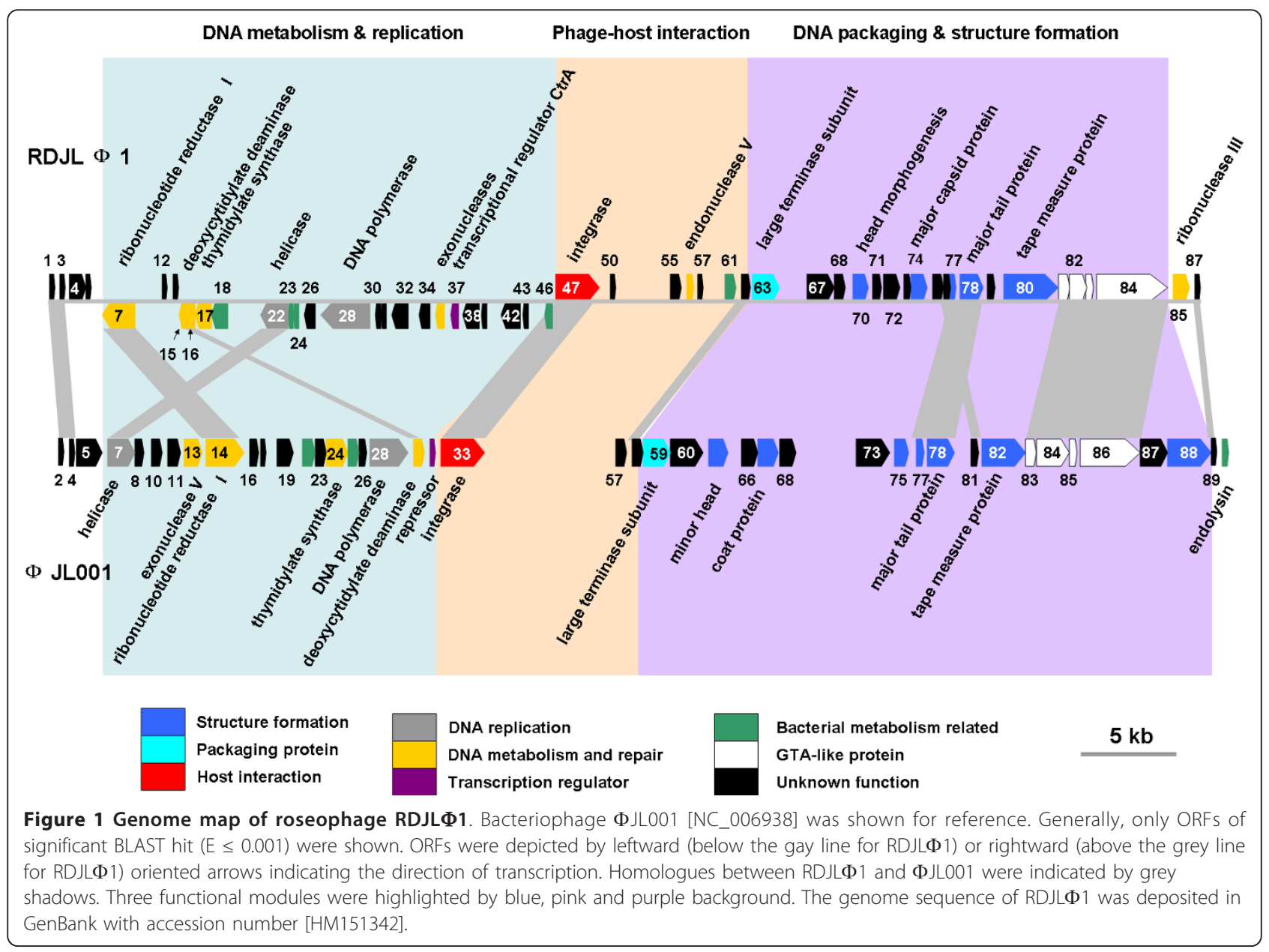


predicted in RDJLФ1 genome, whereas lysis cassettes including four genes (endopeptidase $R z$, embedded $R z 1$, holing and endolysin) were found in YuA and M6 genomes [24]. Phylogenetic analysis based on terminase large subunit (TerL) protein showed that RDJLФ1 fell into the "P22-like headful" cluster and was closely related to Salmonella phages ES18, E1 and Listonella phage $\Phi$ HSIC (Additional file 2). Despite the factor that RDJLФ1, YuA, M6 and ФJL001 share certain genomic similarity, temperate phages YuA, M6 and ФJL001 clustered closely together but distantly to RDJLФ1. Phages that infect marine, even aquatic, bacteria are isolated and characterized less frequently than terrestrial phages, resulting in the difficulty in their taxonomic classifications.

Four ORFs (81, 82, 83 and 84) of RDJL 1 are homologous to RcGTA genes 12,13, 14 and 15, respectively $[12,15]$ (Additional file 1, Figure 2). gp81 is most closely related to the glycoside hydrolase which could also be the putative product of RcGTA gene 12 . ORF 82 was identified as a homologue of RcGTA gene 13 which encodes a structural protein detected by proteomic approaches [26]. ORF 83 contains a phage-related cell wall peptidase domain and was predicted to encode a hydrolase belonging to the NlpC/P60 superfamily. The product of its homologue in RcGTA (gene 14) was not found in proteome [26]. Previously, it was implied that $\mathrm{NlpC} / \mathrm{P} 60$ proteins from bacteriophages may help them penetrate the bacterial cell wall [27]. We propose that gp83 in RDJL $\Phi 1$ may have the same function based on sequence homology. ORF 84 is highly homologous to RcGTA gene 15, the largest gene encoding a single 138 $\mathrm{kDa}$ protein [26], which contains a rhamnosyl transferase homology and was suggested to mediate interaction between RcGTA particles and the capsule of recipient cells [28]. A $147.6 \mathrm{kDa}$ homologous protein was also detected in our previous phage proteome analysis (Table 1). We inferred that this protein serves as a component of tail fibre, which is known to be involved in host specificity in broad types of tailed phages. Typical RcGTA-like gene cluster such as that in $R$. capsulatus is $15 \mathrm{~kb}$ long and encodes 15 gene products (Figure 2) $[12,28]$. Protein sequences of gp81 to gp 84 in RDJLФ1 are $35-49 \%$ identical to the corresponding sequences in RcGTA, which is the highest level of identity that we have found between RcGTA and phage sequences. Similar identity range (30-50\%) was observed between RcGTA and RcGTA-like elements in other alphaproteobacteria [29]. These four RcGTA-like genes have been previously found in phage $\Phi J L 001$ (ORFs 83-86) [12]. It is noteworthy that $\Phi J L 001$ contains unmatched sequences inside ORF84 and 86 when aligned with their homologues (Figure 2, indicated by white boxes internal to blue arrows). This suggests that $\Phi$ JL001 could be relatively distantly related to RcGTA-like elements in alphaproteobacteria.

We retrieved 80 RcGTA-like gene clusters (some are partial) from 77 bacterial genomes and carried out phylogenetic analysis based on the concatenated translated sequences of RcGTA-like genes 12 to 15 . The neighborjoining tree shows that RDJLФ1 are most closely related to Paracoccus denitrificans PD1222, Rhodobacter sphaeroides ATCC 17025 and Rhodobacter sp. SW2 (group II in Figure 3). We also examined the neighboring sequences of all these RcGTA-like structures. Interestingly, typical phage-like genes such as those encoding integrase, small and large terminase subunits, portal, major capsid, and tail tape measure proteins (TMP, coded by tmp) were identified to the left of the RcGTAlike genes from the bacterial genomes of Rhodobacter sp. SW2, Burkholderia phymatum STM815 (plasmid pBPHY01), Variovorax paradoxus S110 and Oligotropha carboxidovorans OM5 (O. carboxidovorans OM5 has two RCGTA-like structures, it refers to "OM5 2" here) (Figure 3). There are three evidences supporting the idea that these structures are not potential RcGTA-like gene clusters but functional elements of prophage genomes. First, no homology to RcGTA-like genes 1 to 11 was found in these gene structures. Second, other phage-like genes were found in the left arms, especially the integrase genes. Third, the tmp genes are much larger than those in RcGTA-like structures (2424 2739 bp vs. $660 \mathrm{bp}$ ). It was demonstrated that phage tail length has significant correspondence with its gene size of tmp [30]. These tmp genes in the aforementioned four bacterial genomes appear to code for components of long phage tails rather than tails of RcGTA-like particles. Lang and Beatty $[12,29]$ suggested that RcGTA-like element is likely a remnant of a prophage ancestor that evolved to be a RcGTA progenitor in an alphaproteobacterium by losing replication, regulatory genes, and that this RcGTA progenitor then have processed a predominantly vertical descent. Partial RcGTA-like gene clusters in genuine phages and putative prophages provide evidence supporting this suggestion. It is interesting that STM815 and S110 are both betaproteobacteria. It is likely that RDJLФ1, ФJL001 and potential prophages in SW2, STM815, S110 and OM5 have been evolving from the proposed "prophage ancestor" and keeping the four conserved RcGTA-like genes, rather than acquired them from RcGTA-like gene clusters in alphaproteobacteria at a recent evolutionary time. They could be the "remnant" of the intermediate form on the way from "prophage ancestor" to RcGTA progenitor. Intriguingly, even a partial RcGTA-like structure and a complete one from the same bacterial genome (e.g. PD1222, ATCC 17025 and OM5) could diverge distantly and evolve independently (Figure 3). 


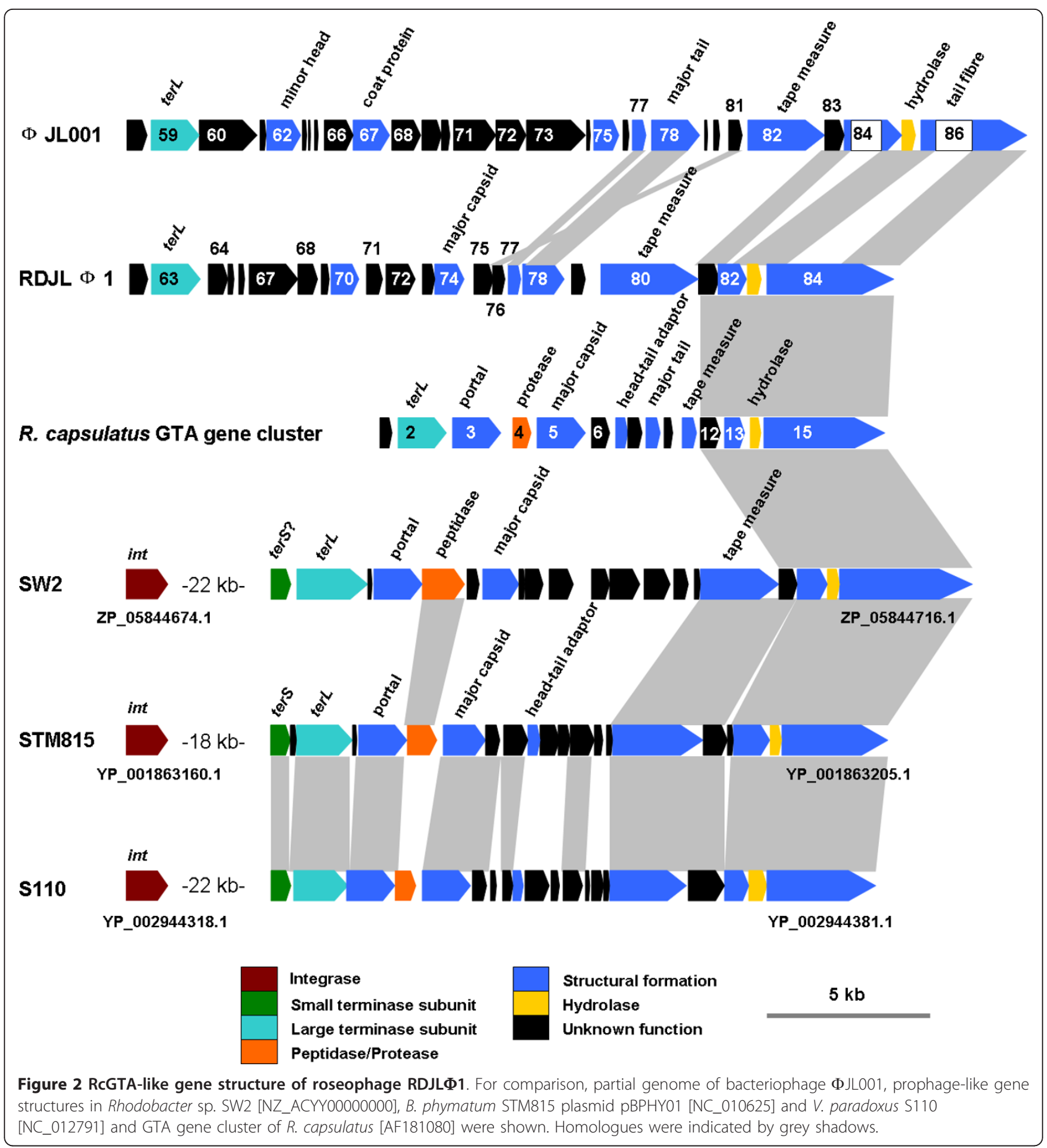

The RcGTA-like gene cluster appears to consist of two modular components: (i) head-to-tail module (gene 1 to 11); (ii) tail fibre and host recognition module (gene 12 to 15). It is interesting that up to date RcGTA-like genes in a (pro)phage have been found either from region (i) or region (ii), but not from both. Likely, the juncture between regions (i) and (ii) is a hotspot for genetic recombination.
The last four RcGTA-like genes seem to be a conserved element as a unit. The vertical descent inside the RcGTA-like gene clusters appears to be unbalanced that losing or keeping some element could be under certain selective pressure. However, the reason why some (pro)phages tend to retain gene module (ii) that is associated with the putative host recognition function is not clear. 


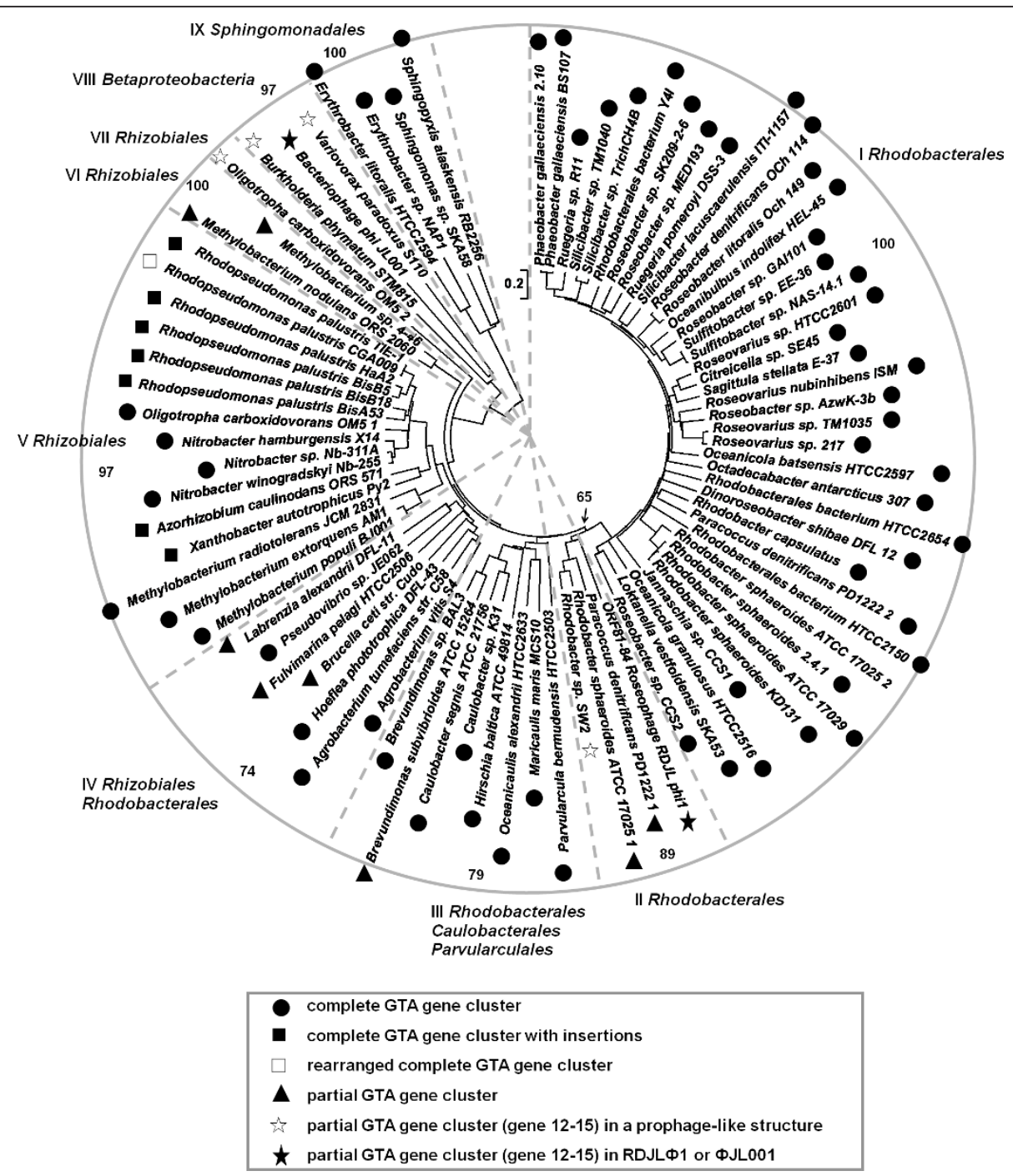

Figure 3 Phylogenetic relationships of concatenated protein sequences of RcGTA-like genes 12-15 from bacteriophages and bacteria. Amino acid sequences were aligned using Clustal X2 [31] and phylogenetic analysis was performed using the Mega 4.0 software [32]. Neighborjoining tree was constructed using the minimum evolution distance with default parameters. Bootstrap resamplings were performed for 1,000 replications and the values (> 50\%) for major branches were shown on the edge of the circular tree, except one in the center indicated by an arrow. Highly supported major subclusters were separated by dashed lines. The numbers in the end of names of bacteria (P. denitrificans PD1222, R. sphaeroides ATCC 17025 and O. carboxidovorans OM5) indicate multiple RcGTA-like elements in one bacterial genome. The four gene clusters in group II only contain RcGTA-like genes 12 to 15. Both PD1222 and ATCC 17025 also contain a complete RcGTA-like gene cluster (in group I). The scale bar represents 0.2 amino acid substitutions per site.

\section{Additional material}

Additional file 1: Genes predicted from Roseophage RDJLФ1 genome.

Additional file 2: Phylogenetic analysis based on the terminase large subunit (TerL) proteins from bacteria and bacteriophages.

\section{Acknowledgements}

We thank Rui Zhang for providing discussion and suggestions in this work. This work was supported by the research programs of MOST

(2007CB815904), NSFC $(40632013,40841023)$ and SOA (200805068) to NJ and Xiamen University 111 Program to FC. YZ was supported by MEL Visiting Fellowship Program (MELRS0931) and NSFC project (41006087).

\section{Author details}

'State Key Laboratory of Marine Environmental Science, Xiamen University, Xiamen 361005, PR China. ${ }^{2}$ Institute of Marine and Environmental Technology, University of Maryland Center for Environmental Science, Baltimore, MD 21202, USA. ${ }^{3}$ Key Laboratory of Urban Environment and Health, Institute of Urban Environment, Chinese Academy of Sciences, Xiamen 361012, PR China.

\section{Authors' contributions}

$\mathrm{YZ}$ isolated the phage, extracted the viral DNA and sequenced the genome. $\mathrm{SH}$ annotated the genome and carried out the phylogenetic and comparative genomic analyses. SH drafted the manuscript, and YZ, FC, NJ 
edited it. NJ and FC organized the study. All authors read and approved the final manuscript.

\section{Competing interests}

The authors declare that they have no competing interests.

Received: 9 August 2010 Accepted: 17 March 2011

Published: 17 March 2011

\section{References}

1. Suttle CA: Viruses in the sea. Nature 2005, 437:356-361.

2. Thingstad TF, Lignell R: Theoretical models for the control of bacterial growth rate, abundance, diversity and carbon demand. Aquat Microb Ecol 1997, 13:19-27.

3. Thingstad TF: Elements of a theory for the mechanism controlling abundance, diversity, and biogeochemical role of lytic bacterial viruses in aquatic systems. Limnol Oceanogr 2000, 45:1320-1328.

4. Weinbauer MG: Ecology of prokaryotic viruses. FEMS Microbiol Rev 2004, 28:127-181.

5. Hendrix RW, Smith MC, Burns RN, Ford ME, Hatfull GF: Evolutionary relationships among diverse bacteriophages and prophages: all the world's a phage. In Proc Natl Acad Sci. Volume 96. USA; 1999:2192-2197.

6. Jiang SC, Paul JH: Seasonal and diel abundance of viruses and occurrence of lysogeny/bacteriocinogeny in the marine environment. Mar Ecol Prog Ser 1994, 104:163-172.

7. Weinbauer MG, Suttle CA: Potential significance of lysogeny to bacteriophage production and bacterial mortality in coastal waters of the Gulf of Mexico. Appl Environ Microbiol 1996, 62:4374-4380.

8. Gonzalez JM, Moran MA: Numerical dominance of a group of marine bacteria in the a-subclass of the class Proteobacteria in coastal seawater. Appl Environ Microbiol 1997, 63:4237-4242.

9. DeLong EF: Microbial community genomics in the ocean. Nat Rev Microbiol 2005, 3:459-469.

10. Newton RJ, Griffin LE, Bowles KM, Meile C, Gifford S, Givens CE, Howard EC, King E, Oakley CA, Reisch CR, Rinta-Kanto JM, Sharma S, Sun S, Varaljay V, Vila-Costa M, Westrich JR, Moran MA: Genome characteristics of a generalist marine bacterial lineage. ISME J 2010, 4:784-798.

11. Chen F, Wang K, Stewart J, Belas R: Induction of multiple prophages from a marine bacterium:a genomic approach. Appl Environ Microbiol 2006, 72:4995-5001.

12. Lang AS, Beatty JT: Importance of widespread gene transfer agent genes in alpha-proteobacteria. Trends Microbiol 2007, 15:54-62.

13. Paul $\mathrm{JH}$ : Prophages in marine bacteria: dangerous molecular time bombs or the key to survival in the seas? ISME J 2008, 2:579-589.

14. Zhao YL, Wang K, Ackermann H, Halden RU, Jiao NZ, Chen F: Searching for a "hidden" prophage in a marine bacterium. Appl Environ Microl 2010, 76:589-595.

15. Marrs B: Genetic recombination in Rhodopseudomonas capsulata. Proc Natl Acad Sci USA 1974, 71:971-973.

16. Rohwer F, Segall A, Steward G, Seguritan V, Breitbart M, Wolven F, Azam F: The complete genomic sequence of the marine phage Roseophage SIO1 shares homology with nonmarine phages. Limnol Oceanogr 2000, 45:408-418.

17. Angly F, Youle M, Nosrat B, Srinagesh S, Rodriguez-Brito B, McNairnie $P$, Deyanat-Yazdi G, Breitbart M, Rohwer F: Genomic analysis of multiple Roseophage SIO1 strains. Environ Microblol 2009, 11:2863-2873.

18. Zhao YL, Wang K, Jiao NZ, Chen F: Genome sequences of two novel phages infecting marine roseobacters. Environ Microbiol 2009, 11:2055-2064.

19. Zhang $Y Y$, Jiao NZ: Roseophage RDJL1, Infecting the Aerobic Anoxygenic Phototrophic Bacterium Roseobacter denitrificans OCh114. Appl Environ Microl 2009, 75:1745-1749.

20. Delcher AL, Harmon D, Kasif S, White O, Salzberg SL: Improved microbial gene identification with GLIMMER. Nucleic Acids Res 1999, 27:4636-4641.

21. Lukashin A, Borodovsky M: GeneMark.hmm: new solutions for gene finding. Nucleic Acids Res 1998, 26:1107-1115.

22. Lowe TM, Eddy SR: tRNAscan-SE: a program for improved detection of transfer RNA genes in genomic sequence. Nucleic Acids Res 1997, 25:955-964.
23. Lohr JE, Chen F, Hill RT: Genomic analysis of bacteriophage ФJL001: insights into its interaction with a sponge-associated alphaproteobacterium. Appl Environ Microbiol 2005, 71:1598-1609.

24. Ceyssens PJ, Mesyanzhinov V, Sykilinda N, Briers Y, Roucourt B, Lavigne R, Robben J, Domashin A, Miroshnikov K, Volckaert G, Hertveldt K: The genome and structural proteome of $\mathrm{YuA}$, a new Pseudomonas aeruginosa phage resembling M6. J Bacterio/ 2008, 190:1429-1435.

25. Kwan T, Liu J, DuBow M, Gros P, Pelletier J: Comparative genomic analysis of 18 Pseudomonas aeruginosa bacteriophages. J Bacteriol 2006, 188:1184-1187

26. Chen F, Spano A, Goodman BE, Blasier KR, Sabat A, Jeffery E, Norris A, Shabanowitz J, Hunt DF, Lebedev N: Proteomic analysis and identification of the structural and regulatory proteins of the Rhodobacter capsulatus gene transfer agent. J Proteome Res 2009, 8:967-973.

27. Anantharaman V, Aravind L: Evolutionary history, structural features and biochemical diversity of the NIpC/P60 superfamily of enzymes. Genome Biol 2003, 4:R11.

28. Lang AS, Beatty JT: Genetic analysis of a bacterial genetic exchange element:the gene transfer agent of Rhodobacter capsulatus. Proc Natl Acad Sci USA 2000, 97:859-864.

29. Lang AS, Beatty JT: Evolutionary implications of phylogenetic analyses of the gene transfer agent (GTA) of Rhodobacter capsulatus. J Mol Evol 2002, 55:534-543.

30. Pedulla ML, Ford ME, Houtz JM, Karthikeyan T, Wadsworth C, Lewis JA, Jacobs-Sera D, Falbo J, Gross J, Pannunzio NR, Brucker W, Kumar V, Kandasamy J, Keenan L, Bardarov S, Kriakov J, Lawrence JG, Jacobs WR, Hendrix RW, Hatfull GF: Origins of highly mosaic mycobacteriophage genomes. Cell 2003, 113:171-182.

31. Larkin MA, Blackshields G, Brown NP, Chenna R, McGettigan PA, McWilliam H, Valentin F, Wallace IM, Wilm A, Lopez R, Thompson JD, Gibson TJ, Higgins DG: ClustalW2 and ClustalX version 2. Bioinformatics 2007, 23:2947-2948.

32. Tamura K, Dudley J, Nei M, Kumar S: MEGA4: Molecular evolutionary genetics analysis MEGA software version 4.0. Mol Biol Evol 2007, 24:1596-1599.

doi:10.1186/1743-422X-8-124

Cite this article as: Huang et al: Complete genome sequence of a marine roseophage provides evidence into the evolution of gene transfer agents in alphaproteobacteria. Virology Journal 2011 8:124.

\section{Submit your next manuscript to BioMed Central and take full advantage of:}

- Convenient online submission

- Thorough peer review

- No space constraints or color figure charges

- Immediate publication on acceptance

- Inclusion in PubMed, CAS, Scopus and Google Scholar

- Research which is freely available for redistribution

Submit your manuscript at www.biomedcentral.com/submit
C) Biomed Central 